Journal of Computer Science 7 (7): 1113-1118, 2011

ISSN 1549-3636

(C) 2011 Science Publications

\title{
Viscous Dissipation Effect on Steady free Convection and Mass Transfer Flow past a Semi-Infinite Flat Plate
}

\author{
${ }^{1}$ Palanisamy Geetha and ${ }^{2}$ Muthamperumal Bhavathikannu Krishna Moorthy \\ ${ }^{1}$ Department of Mathematics, Bannari Amman Institute of Technology, \\ Sathyamangalam, 638 401, India \\ ${ }^{2}$ Department of Mathematics, Institute of Road and Transport Erode, 638 316, India
}

\begin{abstract}
Problem statement: A steady two-dimensional free convection and mass transfer flow past a continuously moving semi-infinite flat plate is studied theoretically, by taking into account the viscous dissipation effect. Approach: The governing equations are transformed into a set of simultaneous ordinary differential equation by using suitable similarity transformations. The coupled differential equations are integrated using the Runge-Kutta Gill method together with the shooting technique. Results: Numerical results were presented for the distribution of velocity, temperature and concentration profiles within the boundary layer. Conclusion: The effects of varying parameter $\mathrm{Gb}$, the Gebhart number, Sc, Schmidt number and Pr, Prandtl number on the velocity, temperature and concentration profiles were displayed graphically for different values of parameters entering into the problem. Significant changes were observed in heat and mass transfer coefficient, due to viscous dissipation in the medium. In addition, the skin-friction coefficient, Nusselt number and Sherwood number were shown in a tabular form.
\end{abstract}

Key words: Heat transfer, mass transfer, moving surface, prandtl number, gebhart number, schmidt number, viscous dissipation, skin-friction coefficient, sherwood number

\section{INTRODUCTION}

Owing to their numerous applications in industrial manufacturing process, the problem of heat and mass transfer in the boundary layers of a continuously moving semi-infinite flat plate has attracted the attention of researchers for the past 3 decades. Some of the application areas are hot rolling, study production, metal spinning, drawing plastic films, glass blowing, continuous casting of metals and spinning of fibers. Annealing and thinning of copper wire is another example. In all these cases, the quality of the final product depends on the rate of heat and mass transfer at the moving surface. By drawing the strips in an electrically conducting fluid subjected to a magnetic field the rate of cooling can be controlled and the final products of desired characteristic might be achieved.

Flow in the boundary layer of a viscous fluid on a moving continuous solid surfaces was investigated by Sakiadis (1961a). It was observed that the boundary layer growth is in the direction of motion of the continuous solid surface and is different from that of the Blasius flow past a flat plate. Still the boundary layer theory and the basic differential equations are applicable. Many authors have attacked this problem, however, this attack has been limited to some constrains on the surface velocity and temperature distribution. In addition to these investigations, experimental and theoretical studies of the flow and temperature fields in the boundary layer on a continuous moving surface have been made by Tsou et al. (1967) for different values of the Prandtl number. Measure of the laminar velocity field were in excellent agreement with the analytical predictions. The heat transfer of above problem of different physical situations have been studied. Viscous dissipation which, appears as a source term in the fluid flow generates appreciable temperature, gives the rate at which mechanical energy is converted into heat in a viscous fluid per unit volume. This effect is of particular significant in natural convection in various devices that are subjected to large variation of gravitational force or that operate at high rotational speeds, pointed by Gebhart (1962) in his study of viscous dissipation on natural convection in fluids. Similarity solutions for the same problem with exponential variation of wall

Corresponding Author: P. Geetha, Department of Mathematics Bannari Amman Institute of Technology,

Sathyamangalam, 638 401, India 


\section{J. Computer Sci., 7 (7): 1113-1118, 2011}

temperature was obtained by Gebhart and Mollendorf (1969). It is observed that the effect of viscous dissipation is more predominant in vigorous natural convection and mixed convection processes. They also have shown the existence of similarity solution for the external flow over an infinite flat vertical surface having an exponential variation of surface temperature. Vajravelu and Hadjinicolaou (1993) studied the heat transfer characteristic in the laminar boundary layer of a viscous fluid over a linearly stretching surface with variable wall temperature subject to suction or blowing. They considered the effects of viscous dissipation and internal heat generation. The effect of chemical reaction, heat and mass transfer on a laminar flow along a semi infinite horizontal plate have been studied by Anjali and Kandasamy (1999). Studied the effect of viscous dissipation on heat transfer in flow past a continuously moving semi-infinite flat plate. Natural convection boundary layer flow over a sphere of a viscous incompressible electrically conducting fluid in the presence of magnetic field and heat generation with the effects of viscous dissipation has been investigated by Alam et al. (2007) and Subhas Abel et al. (2011) studied the effect of viscous and Joules dissipation on MHD flow over a porous nonlinear vertical stretching sheet with partial slip. Since the pioneering work of Sakiadis (1961b) which studied the moving plate flow problem, wherein various aspects of the problem have been investigated by Cortell (2006). Cortell (2007a) has worked on viscous flow and heat transfer over a nonlinearly stretching sheet. Cortell (2008) further investigated on the effects of viscous dissipation and radiation on the thermal boundary layer, over a nonlinear stretching sheet. Moreover, Cortell (2007b) studied the viscous flow and heat transfer over a nonlinearly stretching surface. Raptis and Perdikis (2006) studied viscous flow near a non-linear stretching sheet in the presence of a chemical reaction and magnetic field. Kumar (2009) investigated the study of radiation and viscous dissipation effects over a stretching surface subjected to variable heat flux in presence of transverse magnetic field. Kishan and Amrutha (2010) studied the two-dimensional steady nonlinear MHD boundary layer flow of an incompressible, viscous, electrically conductive and Boussinesq fluid flowing over a vertical stretching surface in the presence of uniform magnetic field by taking into account the viscous dissipation with heat, mass transfer chemical reaction and thermal stratification effects. A study on MHD free convective flow of an incompressible viscous dissipative fluid in an infinite vertical oscillating plate with constant heat flux has been carried by Kishan et al. (2006). Kairi et al. (2011) investigated the combined effect of viscous dissipation and radiation on natural convection in nonDarcy porous medium saturated with non-Newtonian fluid of variable viscosity. El-Arabawy (2009) studied the effects of suction/injection and chemical reaction on mass transfer over a stretching surface. Adegbie and Alao (2007). investigated the steady-state flow of Newtonian liquid with exponential temperaturedependent viscosity and substantial viscous heat generation between symmetrically parallel heated walls with walls at different temperatures.

The aim of the present study is to study the effect of viscous dissipation on heat transfer and mass transfer in flow past a continuously moving semi-infinite flat plate. The analysis showed that the viscous dissipation have significant influence on the non-dimensional heat and mass transfer coefficients.

Mathematical formulation: Consider the twodimensional, flow past a continuously moving semiinfinite flat plate. The $\mathrm{x}$-axis is assumed to be taken along the plate and the y-axis normal to the plate. u,v are the velocity components in the $\mathrm{x}$ and $\mathrm{y}$ directions. Now, the governing equations under the boundary layer and Boussinesq approximations may be written as:

$$
\begin{aligned}
& \frac{\partial u}{\partial x}+\frac{\partial v}{\partial y}=0 \\
& u \frac{\partial u}{\partial x}+v \frac{\partial u}{\partial y}=v \frac{\partial^{2} u}{\partial y^{2}} \\
& u \frac{\partial T}{\partial x}+v \frac{\partial T}{\partial y}=\frac{k}{\rho c_{p}} \frac{\partial^{2} T}{\partial y^{2}}+\frac{v}{c_{p}}\left(\frac{\partial u}{\partial y}\right)^{2} \\
& u \frac{\partial C}{\partial x}+v \frac{\partial C}{\partial y}=D \frac{\partial^{2} C}{\partial y^{2}}
\end{aligned}
$$

Along with the boundary conditions:

$$
\begin{aligned}
& \mathrm{u}=\mathrm{U}_{0} ; \mathrm{v}=0 ; \quad \mathrm{T}=\mathrm{T}_{\mathrm{w}} ; \quad \mathrm{C}=\mathrm{C}_{\mathrm{w}} \text { at } \mathrm{y}=0 \\
& \mathrm{u}=0 ; \mathrm{T} \rightarrow \mathrm{T}_{\infty} ; \mathrm{C} \rightarrow \mathrm{C}_{\infty} \text { at } \mathrm{y} \rightarrow \infty
\end{aligned}
$$

Here:

$\mathrm{a}=$ Thermal diffusivity constant 
$\mathrm{c}_{\mathrm{p}}=$ Specific heat

$\mathrm{v}=$ Kinematic viscosity of the ambient fluid

The above set of partial differential equations are converted into ordinary differential equations using the following similarity transformation:

$\eta=y \sqrt{\frac{U_{0}}{v x}}$

$\psi(\mathrm{x}, \mathrm{y})=\sqrt{v \mathrm{vU}_{0}} \mathrm{f}$

$\theta=\frac{T-T_{\infty}}{T_{w}-T_{\infty}}$

$\varphi=\frac{C-C_{\infty}}{C_{w}-C_{\infty}}$

Where:

$\eta=$ The similarity variable

$\theta=$ The dimensionless stream function depends on $\eta$ only

Let $\psi$ be the stream function defined such that $\mathrm{u}=\frac{\partial \psi}{\partial \mathrm{y}}$ and $\quad \mathrm{v}=-\frac{\partial \psi}{\partial \mathrm{x}}$

so that it automatically

satisfies the continuity Eq. 1. The final transformed equation and boundary conditions are:

$f^{\prime \prime \prime}+\frac{1}{2} \mathrm{ff}^{\prime \prime}=0$

$\theta^{\prime \prime}+\frac{1}{2} \operatorname{Prf} \theta^{\prime}+\mathrm{Gb} \operatorname{Pr}\left(\mathrm{f}^{\prime \prime}\right)^{2}=0$

$\phi^{\prime \prime}+\frac{\mathrm{Sc}}{2} \mathrm{f} \phi^{\prime}=0$

With the boundary and initial conditions as:

$\mathrm{f}(0)=0 ; \mathrm{f}^{\prime}(0)=1 ; \theta(0)=1 ; \phi(0)=1 \quad$ at $\eta=0$

$\mathrm{f}^{\prime}(\infty)=0 ; \quad \theta(\infty)=0 ; \quad \phi(\infty)=0 \quad$ at $\eta \rightarrow \infty$
The non-dimensional numbers are defined as the viscous dissipation parameter known as the Gebhart number, given by $G b=\frac{U^{2}}{c_{p}\left(T_{w}-T_{\infty}\right)}$, Schmidt number given by $\mathrm{Sc}=\mathrm{v} / \mathrm{D}$ and the Prandtl number given by $\operatorname{Pr}=$ v/a.

\section{MATERIALS AND METHODS}

The set of Eq. 10-12 together with the boundary conditions (11) have been solved numerically by applying shooting technique along with Runge-Kutta Gill method .From the process of numerical computation, the skin-friction coefficient, the local Nusselt number and the local Sherwood number, which are respectively proportional to $\mathrm{f}^{\prime \prime}(0),-\theta^{\prime}(0)$ and $\phi^{\prime}(0)$ are also sorted out and their numerical values are presented in a tabular form.

The parameters for the present problem are the local Nusselt number and the local Sherwood number, which indicate physically the rate of heat transfer and the rate of mass transfer respectively.

Now the heat flux $\left(\mathrm{q}_{\mathrm{w}}\right)$ and the mass flux $\left(\mathrm{M}_{\mathrm{w}}\right)$ at the wall are given by:

$\mathrm{q}_{\mathrm{w}}=-\mathrm{k}\left(\frac{\partial \mathrm{T}}{\partial \mathrm{y}}\right)_{\mathrm{y}=0}=-\mathrm{k}\left(\mathrm{T}_{\mathrm{w}}-\mathrm{T}_{\infty}\right) \sqrt{\frac{\mathrm{U}_{0}}{v \mathrm{x}}} \theta^{\prime}(0)$

And:

$\mathrm{M}_{\mathrm{w}}=-\mathrm{D}\left(\frac{\partial \mathrm{C}}{\partial \mathrm{y}}\right)_{\mathrm{y}=0}=-\mathrm{D}\left(\mathrm{C}_{\mathrm{w}}-\mathrm{C}_{\infty}\right) \sqrt{\frac{\mathrm{U}_{0}}{\mathrm{vx}}} \varphi^{\prime}(0)$

Hence the Nusselt number $(\mathrm{Nu})$ and Sherwood number (Sh) are obtained as:

$$
\begin{aligned}
& \mathrm{Nu}=\frac{\mathrm{xq}_{\mathrm{w}}}{\mathrm{k}\left(\mathrm{T}_{\mathrm{w}}-\mathrm{T}_{\infty}\right)}=-(\mathrm{Re})^{\frac{1}{2}} \theta^{\prime}(0) \\
& \text { i.e. } \mathrm{Nu}(\mathrm{Re})^{-\frac{1}{2}}=-\theta^{\prime}(0) \text { and } \\
& \mathrm{Sh}=\frac{\mathrm{xM}_{\mathrm{w}}}{\mathrm{D}\left(\mathrm{C}_{\mathrm{w}}-\mathrm{C}_{\infty}\right)}=-(\mathrm{Re})^{\frac{1}{2}} \varphi^{\prime}(0) \\
& \text { i.e. } \mathrm{Sh}(\mathrm{Re})^{-\frac{1}{2}}=-\varphi^{\prime}(0)
\end{aligned}
$$

where, $\operatorname{Re}=\mathrm{U}_{0} \mathrm{x} / \mathrm{v}$ is the Reynold's number.

The ordinary differential Eq. 10-12 along the boundary condition Eq. 13 are solved by giving approximate initial guess values for the missing initial conditions of $f(0), \theta^{\prime}(0), \phi^{\prime}(0)$ and these values are matched with the corresponding boundary conditions at $\mathrm{f}^{\prime}(\infty) \theta(\infty)$ and $\phi(\infty)$. Extensive calculations have been 
performed to obtain the flow and temperature fields for a wide range of parameters $0<\operatorname{Pr} \leq 10,0<\mathrm{Sc} \leq 1$ and $1 \leq \mathrm{Gb} \leq 10$.

\section{RESULTS}

Table 1 shows numerical values of viscous dissipation effects on $\mathrm{f}^{\prime \prime}(0), \theta^{\prime}(0)$ and $\phi^{\prime}(0)$.

The profiles for velocity, temperature and concentration are shown in Fig. 1-7 respectively with various values of the parameters.

\begin{tabular}{llllrl}
\multicolumn{5}{c}{ Table 1: Viscous dissipation effects on $\mathrm{f}^{\prime \prime}(0), \theta^{\prime}(0)$ and $\phi^{\prime}(0)$} \\
\hline Sc & $\operatorname{Pr}$ & $\mathrm{Gb}$ & $\mathrm{f} "(0)$ & \multicolumn{1}{c}{$\theta^{\prime}(0)$} & $\phi^{\prime}(0)$ \\
\hline 0.3 & 0.71 & 1 & -0.4439 & -0.1894 & -0.2183 \\
& & 5 & -0.4439 & 0.4710 & -0.2490 \\
& & 10 & -0.4439 & 1.2966 & -0.2873 \\
0.6 & 7 & 1 & -0.4485 & -0.2068 & -0.3268 \\
& & 5 & -0.4485 & 4.0156 & -0.5455 \\
& & 10 & -0.4485 & 9.4169 & -0.8166 \\
0.78 & 10 & 1 & -0.4539 & -0.2270 & -0.3910 \\
& & 5 & -0.4539 & 5.5742 & -0.6939 \\
& & 10 & -0.4539 & 12.8260 & -1.0723 \\
\hline
\end{tabular}

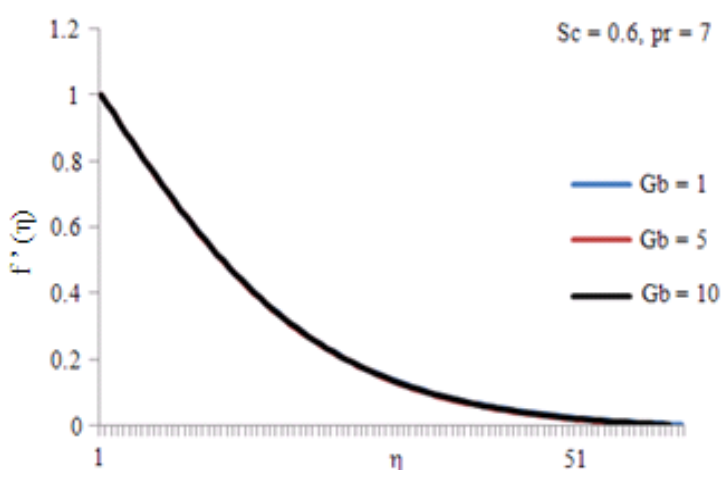

Fig. 1: Effect of viscous dissipation parameter $\mathrm{Gb}$ on non-dimensional velocity $f^{\prime}$

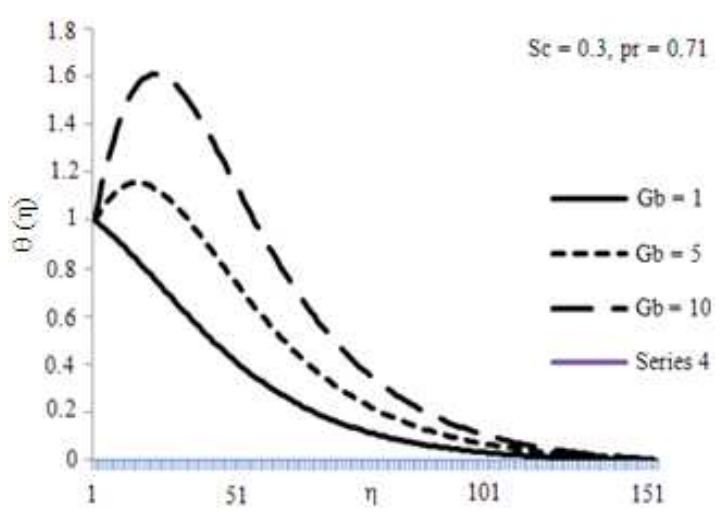

Fig. 2: Effect of viscous dissipation parameter $\mathrm{Gb}$ on non-dimensional temperature $\theta$

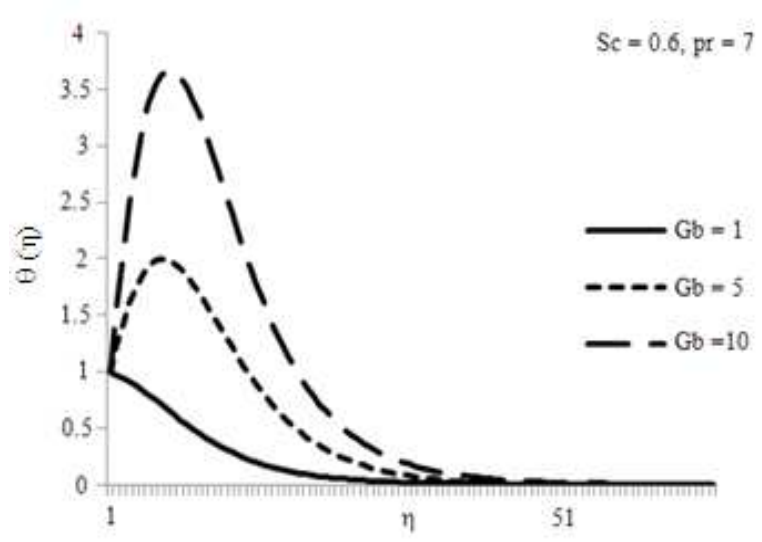

Fig. 3: Effect of viscous dissipation par $\theta$

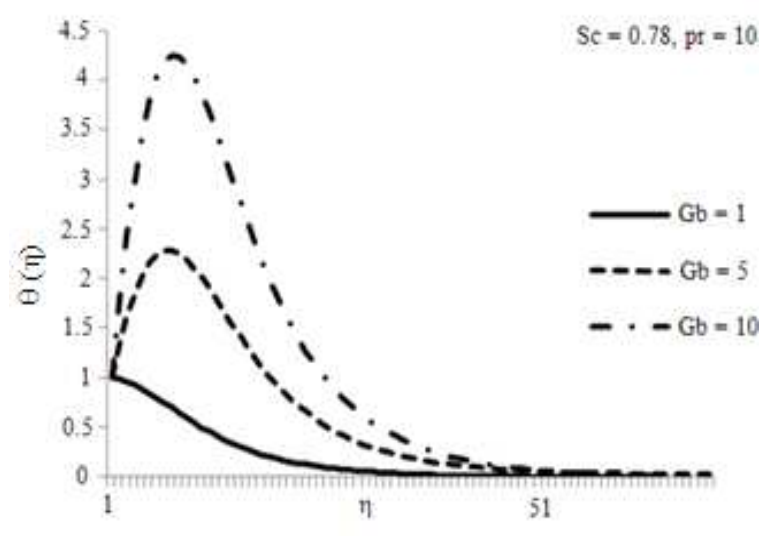

Fig. 4: Effect of viscous dissipation parameter $\mathrm{Gb}$ on non-dimensional temperature $\theta$

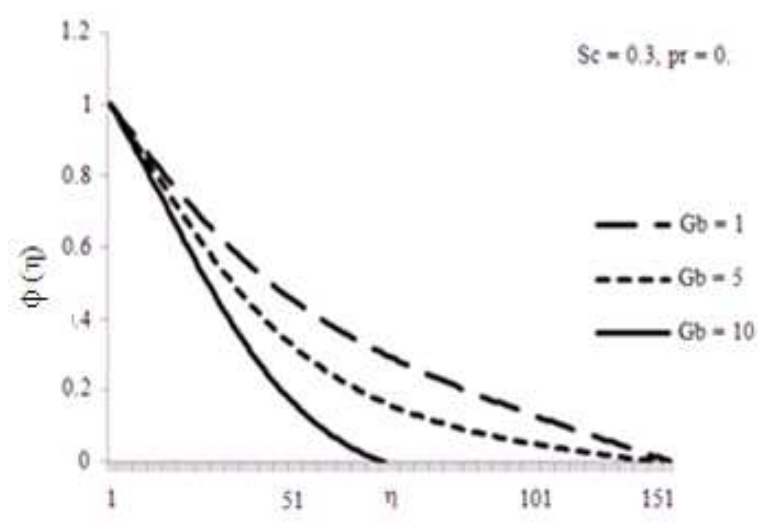

Fig. 5: Effect of viscous dissipation parameter $\mathrm{Gb}$ on non-dimensional concentration $\phi$ 


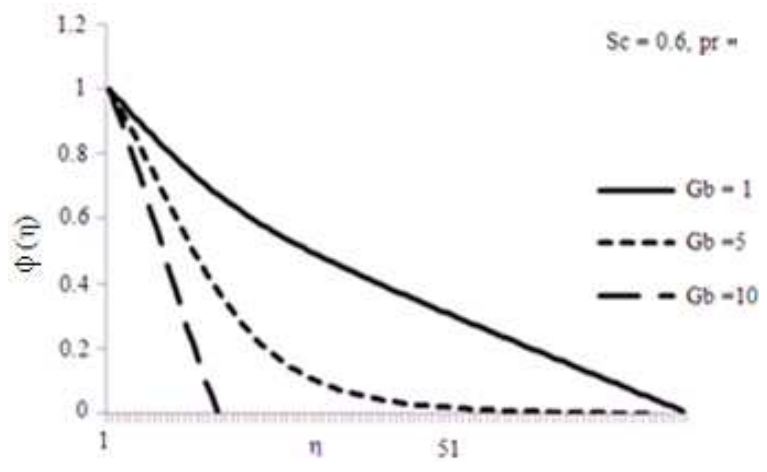

Fig. 6: Effect of viscous dissipation parameter $\mathrm{Gb}$ on non-dimensional concentration $\phi$

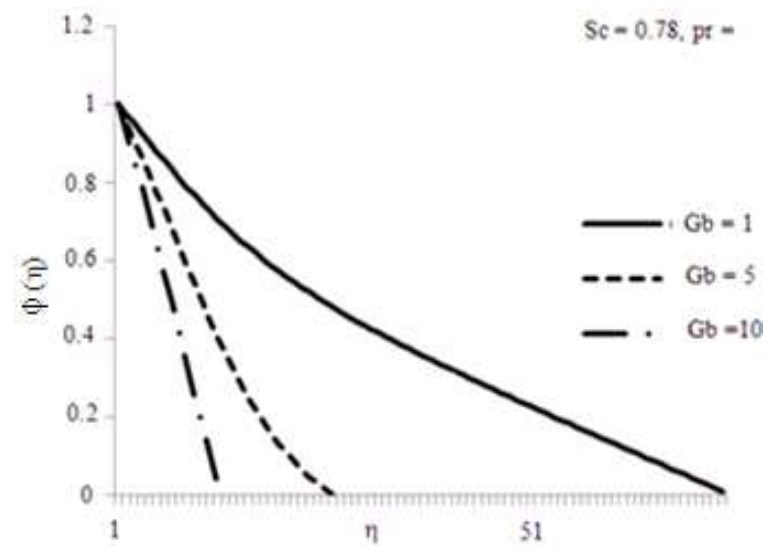

Fig. 7: Effect of viscous dissipation parameter $\mathrm{Gb}$ on non-dimensional concentration $\phi$

\section{DISCUSSION}

Now we discuss the results. The velocity, temperature and concentration profiles in the boundary layer are plotted for selected values of the governing parameters. The non-dimensional velocity across the boundary layer is plotted for varying values of $\mathrm{Gb}$ $(1,5,10)$ with Prandtl number $\operatorname{Pr}=0.71,7,10$ and Schmidt number Sc $=0.3,0.6,0.78$ to depict the flow field. The magnitude of the velocity remains same as the value of the flow governing parameter increases which is shown in Fig. 1.

The non-dimensional temperature distribution inside the boundary layer are presented in Fig. 2-4. The thermal boundary layer thickness is increased with the increasing of $\mathrm{Gb}$.

The non-dimensional concentration distribution inside the boundary layer are presented in Fig. 5-7. The concentration decreases with the increase of $\mathrm{Gb}$.
The non-dimensional heat transfer coefficient and mass transfer coefficient are plotted against the parameter $\mathrm{Gb}$. The heat transfer coefficient and mass transfer coefficient are increased with the Prandtl number and also as the value of the flow governing parameter is increased.

Table 1 presents the effect of $\mathrm{Sc}, \mathrm{Pr}$ and $\mathrm{Gb}$ on the skin friction coefficient $\mathrm{f}^{\prime}$ ' $(0)$, the Nusselt number $-\theta^{\prime}(0)$ and the Sherwood number - $\phi^{\prime}(0)$. The results reveal that the Nusselt number and the Sherwood number increases as $\mathrm{Sc}, \mathrm{Pr}$ and $\mathrm{Gb}$ increases.

\section{CONCLUSION}

An increase in the viscous dissipation parameter $\mathrm{Gb}$ resulted:

- An increase in temperature

- A fall in concentration

- An increase in heat transfer coefficient and mass transfer coefficient

\section{ACKNOWLEDGEMENT}

The research render their heartfelt thanks to Dr. T. Govindarajulu former Professor and Head of Department of Mathematics, Anna University, Chennai for his generous help. The authors wish to thank the Director IRT Chennai and the Principal IRTT, Erode for their generous help. The authors wish to thank the Chairman, Director, Chief Executive, Principal, Bannari Amman Institute of Technology for their support to do this study.

\section{REFERENCES}

Adegbie, K.S. and F.I. Alao, 2007. Flow of temperature-dependent viscous fluid between parallel heated walls: Exact analytical solutions in the presence of viscous dissipation. J. Math. Stat., 3: 12-14. DOI: 10.3844/JMSSP.2007.12.14

Alam, M.M., M.A. Alim and M.M.K. Chowdhury, 2007. Viscous dissipation effects on MHD natural convection flow over a sphere in the presence of heat generation. Nonlinear Analysis: Model. Control, $\quad$ 12: 447-459. http://www.lana.1t/journal/27/MdM_Alam.pdf

Anjali, S.P. and R. Kandasamy, 1999. Effects of chemical reaction, heat and mass transfer on laminar flow along a semi infinite horizontal plate. Heat. Mass Trans. 35: 465-467. DOI: $10.1007 / \mathrm{S} 002310050349$ 


\section{J. Computer Sci., 7 (7): 1113-1118, 2011}

Cortell, R., 2006. Effects of viscous dissipation and work done by deformation on the MHD flow and heat transfer of a viscoelastic fluid over a stretching sheet. Phys. Lett. A, 357: 298-305. DOI: 10.1016/J.PHYSLETA.2006.04.051

Cortell, R., 2007a. MHD flow and heat transfer of an electrically conducting fluid of second grade in a porous medium over a stretching sheet subject with chemically reactive species. Chemical Eng. Process., $\quad 46$ : 721-728. DOI: 10.1016/J.CEP.2006.09.008

Cortell, R., 2007b. Viscous flow and heat transfer over a non-linearly stretching sheet. Applied Math. Comput., 184: 864-873. DOI: 10.1016/J.AMC.2006.06.077

Cortell, R., 2008. Effects of viscous dissipation and radiation on the thermal boundary layer over a nonlinearly stretching sheet. Phys. Lett. A, 372: 631636. DOI: 10.1016/J.PHYSLETA.2007.08.005

El-Arabawy, H.A.M., 2009, Exact solution of mass transfer over a stretching surface with chemical reaction and suction/injection. J. Math. Stat., 5: 159-166. DOI: 10.3844/JMSSP.2009.159.166

Gebhart B., 1962. Effect of viscous dissipation in natural convection. J. Fluid. Mech., 14: 225-232. DOI: $10.1017 /$ S0022112062001196

Gebhart, B. and Mollendorf, 1969. Viscous dissipation in external natural convection flows. J. Fluid. Mech., 38: 97-107. DOI: 10.1017/S0022112069000061

Kairi, R.R., P.V.S.N. Murthy and C.O. Ng, 2011. Effect of viscous dissipation on natural convection in a non-darcy porous medium saturated with nonnewtonian fluid of variable viscosity. Open Transport Phenomena J., 3. 1-8. DOI: 10.2174/1877729501103010001

Kishan, N. and P. Amrutha, 2010. Effects of viscous dissipation on MHD flow with heat and mass transfer over a stretching surface with heat source, thermal stratification and chemical reaction. J. Naval Archit. Marine Eng., 7 11-18. DOI: 10.3329/jname.v7il.3254

http://www.banglajol.info/index.php/JNAME/articl e/view/3254
Kishan. N., Srihari and Rao, J.A., 2006. MHD free convective flow of an incompressible viscous dissipative fluid in an infinite vertical oscillating plate with constant heat flux. J. Energy Heat Mass Trans., 28: 19-28. http://direct.bl.uk/bld/PlaceOrder.do?UIN=200051 073\&ETOC $=$ RN\& from $=$ searchengine

Kumar, H., 2009. Radiative heat transfer with hydromagnetic flow and viscous dissipation over a stretching surface in the presence of variable heat flux. Thermal Sci., 13: 163-169. DOI: 10.2298/TSCI0902163K

Raptis, A. and C. Perdikis, 2006. Viscous flow over a non-linearly stretching sheet in the presence of a chemical reaction and magnetic field. Int. J. NonLinear Mech., 41: 527-529. DOI: DOI: 10.1016/J.IJNONLINMEC.2005.12.003

Sakiadis, B.C., 1961a. Boundary layer behavior on continuous moving solid surfaces i. boundary layer equations for two-dimensional and axi-symmetric flow. AIChE J., 7: 26-28. DOI: 10.1002/aic.690070108

Sakiadis, B.C., 1961b. Boundary layer behavior on continuous solid surfaces: II the boundary layer on a continuous flat surfaces. AIChE J., 7: 221-225. DOI: 10.1002/aic.690070211

Subhas Abel. M., K.A. Kumar and R. Ravikumara, 2011. MHD flow and heat transfer with effects of buoyancy, viscous and joules dissipation over a nonlinear vertical stretching porous sheet with partial slip. Sci. Res., 3: 285-291. DOI: 10.4236/eng.2011.33033

Tsou, F.K., E.M. sparrow and R.J. Goldstein, 1967. Flow and heat transfer in the boundary layers on a continuous moving surface. Int. J. Heat Mass Trans., 10: 219-235. DOI: 10.1016/00179310(67)90100-7

Vajravelu, K. and A. Hadjinicalaou, 1993. Heat transfer in a viscous fluid over a stretching sheet with viscous dissipation and internal heat generation. Int. Comm. Heat. Mass Trans., 20: 417-430. DOI: 10.1016/0735-1933(93)90026-R 